\title{
Numerical Simulation of a Single-Phase Flow Through Fractures with Permeable, Porous and Non-Ductile Walls
}

\author{
Nader Pour Mahmoud \\ Mechanical Engineering Department \\ Urmia University \\ Urmia, Iran \\ N.pormahmod@urmia.ac.ir
}

\author{
Aidin Zabihi \\ Mechanical Engineering Department \\ Urmia University \\ Urmia, Iran \\ St_a.zabihi@urmia.ac.ir
}

\begin{abstract}
This paper attempts to study flows within fractures through a set of numerical simulations. In addition, a special care is given to hydraulic features and characteristics of fractures. The research is performed through the application of calculative fluid dynamics and a finite volume discrete schema. The investigated flows are laminar, single-phase and stable flows of water and air through fractures with penetrable walls. The selected fracture geometry is inspired from the tomographic scan of a stone fracture. Water and air are modeled in fractures with permeable walls and different permeability levels. It has been observed that in case of permeable matrixes, the friction coefficient is lower compared to impermeable matrixes. In fact permeability reduced friction. In addition, highest pressure drops were observed in areas with smaller fracture diaphragms. Nonetheless, the surrounding area of the fracture is analyzed with the consideration of Darcy's rule.
\end{abstract}

Keywords-fracture; pressure gradient; porous media; singlephase flow numerical simulation; Darcy's rule; laminar flow; matrix

\section{INTRODUCTION}

Fractures of earth crust are in different scales. These fractures are significant for certain fields of study including geology, oil engineering and mechanical engineering as well. Fractures provide an easy route for the flow of fluid and facilitate the flow through barriers throughout the whole fracture and act as a hydraulic conductor. The amount of carbon dioxide present in atmosphere has significantly increased during the past few years. This high level of carbon dioxide results in ground warming. Underground brines have the highest capacity for storage of carbon dioxide. Underground brine aquifers are made of porous stones with low permeability. There are also fractures on these stones that can act as natural fluid pipelines. Therefore, carbon dioxide can be stored in these underground structures. It is assumed that fractured areas in reservoirs are of a lower permeability. Considering the low surface tension underground or undersurface fluids, it can be concluded that carbon dioxide is stored in these reservoirs with a low viscosity. Several experimental studies have been performed regarding the contexts of a single-fracture [1], flows within intersecting fractures [2], and flows through fractured porous environments [3]. The present study analyzes the flow through a fracture and evaluates the flow rate for different types of pressure drops and various permeability levels for matrixes of fracture surroundings. It is worth mentioning that Navier-Stokes nonlinear equations have been used for expression of behavior of flows through fractures [4]. Several models have been proposed for the investigation of flows through porous environments and fractures [5]. More recently, the behavior of flow through rough fractures was studied. In addition, other researchers have investigated the behavior of flow of fluids through real fractures through the application of the finite differences formula [6]. The aforementioned researchers have shown that in fractures, flow behavior depends on twists and roughness of fractures. Authors in [7] used the 2TOUGH code for analysis of non-saturated flows within fractured stones. They concluded that the method of continuity is suitable. In addition, their simulations revealed that matrix permeability can have a significant effect on behavior of flow traffic behavior. Authors in [8] investigated the flow and transfer of salts around an injection well with a growing crack. Authors in [9] measured the surfaces of stone fracture through the application of 3D laser scan method. This study investigates the reliability of fractal models for fracture geometry.

In this regard, a theoretical fracture model was developed and the results were compared with those obtained from experimental observations. Authors in [10], used the Brazilian test method for a fractured layered Brea sand stone with a length of 10.15 centimeters and width of 2.64 centimeters. It is worth mentioning that the overall dimensions of experimental sample were 10.2 by 2.7 by 0.77 centimeters. In addition a high quality CT-scan and 240 cubic micrometers Voxel were used. The present study elaborates on simulation of a single-phase flow through a fracture with permeable surrounding. The geometry under investigation is obtained from a CT-Scan slice of Brea sand stone prepared by [10]. Authors in [11] calculated the friction coefficient for fractures with impermeable walls. 
Numerical simulations of the present study have been performed through solvation of Navier-Stokes equations through the application of CFD Ansys Fluent. In addition it must be stated that similar flow rates have been calculated for various pressure gradients.

A fracture surrounding permeability rate of 2-2000 md has also been selected for both flows of water and air. Results obtained regarding the fluid flow of water have been compared with those obtained by other researchers. Results of this simulation have several applications in reservoirs with constant pressure and several fractures. The following explains the manners of modeling of flow, and simulation of marginal condition of the flow [12]. Afterwards, results of simulations are expressed and ultimately, the mentioned results are compared with results obtained from earlier research works.

\section{EQUATIONS GOVERNING THE FLOW}

Simulation of a flow using the Stokes equation is a relatively easy task and requires significantly fewer calculations compared with the full Navier-Stokes formula. However, the Stokes equation is only applicable to laminar flows. We have modeled the equations of flow of fluids through fractures through numerical solutions. The physical principles of flow through fracture can be defined using the mass and momentum related equations. However, the movement equations of a single-phase Newtonian and incompressible fluid flow can be written as follows:

$$
\begin{gathered}
\partial u / \partial x+\partial v / d y+\partial w / \partial x=0 \\
\rho\left(\frac{\partial u}{\partial t}+u \frac{\partial u}{\partial x}+v \frac{\partial u}{\partial y}+w \frac{\partial u}{\partial z}\right)=-\frac{\partial p}{\partial x}+\mu\left(\frac{\partial^{2} u}{\partial x^{2}}+\frac{\partial^{2} u}{\partial y^{2}}+\frac{\partial^{2} u}{\partial z^{2}}\right) \\
\rho\left(\frac{\partial v}{\partial t}+u \frac{\partial v}{\partial x}+v \frac{\partial v}{\partial y}+w \frac{\partial v}{\partial z}\right)=-\frac{\partial p}{\partial y}+\mu\left(\frac{\partial^{2} v}{\partial x^{2}}+\frac{\partial^{2} v}{\partial y^{2}}+\frac{\partial^{2} v}{\partial z^{2}}\right) \\
\rho\left(\frac{\partial w}{\partial t}+u \frac{\partial w}{\partial x}+v \frac{\partial w}{\partial y}+w \frac{\partial w}{\partial z}\right)=-\frac{\partial p}{\partial z}+\mu\left(\frac{\partial^{2} w}{\partial x^{2}}+\frac{\partial^{2} w}{\partial y^{2}}+\frac{\partial^{2} w}{\partial z^{2}}\right)
\end{gathered}
$$

In the upper equations, expressions of $\mathrm{u}, \mathrm{v}$ and $\mathrm{w}$ are respectively the components of velocity in respective order of $\mathrm{x}, \mathrm{y}$ and $\mathrm{z}$ directions. Equation (1), is the continuity equation and equations (2), (3) and (4) are the Navier-Stokes equations that express the movement of an incompressible fluid in a three dimensional figure. The hypothesis of incompressibility of fluids (for example Water, Oil, Gas and Air) under the surface of crust is already accepted. By the use of steady state form of Navier-Stokes equation, the flow of fluid can be rewritten in the following vector form:

$$
\rho(u . \nabla) u=\mu \nabla^{2} u-\nabla p
$$

The upper equation is a differential equation with partial derivatives and no general solution in a way that, several hypothesis need to be considered in order to be able to analytically solve it. Hypothesizing a steady state with no compressibility will not give us a general solution because the equation is still non-linear. The main problem in solvation of the upper equation is presence of the $\rho(u . \nabla) u$ term. In certain cases, this term is minimal while modeling for undersurface flows. In these cases, this term can be neglected. When this term is removed from the Navier-Stokes equation, the equation gains a high level of simplicity. Therefore it becomes easily solvable. Laminar and dense flows are examples of models of fluid movement that can be expressed by mathematical linear equation of Stokes.

$$
\mu \nabla^{2} u=\nabla p
$$

The upper equation can be used for simulation of flows through fractures; however in case of fractures with parallel walls. It is also a very suitable approximation for extremely laminar flows even when the fracture walls are not perfectly parallel. In terms of low velocities, the importance of the upper term is signified when the geometry of fracture has bended or there are sharp corners or when the goal is analysis of a suitably scaled flow near the wall of fracture with high coarseness frequency. The method of calculative fluid dynamic (CFD) namely as Ansys Fluent, is made use of in order to numerically solve the steady state Navier-Stokes equation. These equations are solved through the finite volume method based on transformation of governing mathematical equations into algebraic equations which are solved numerically. The fractured stone sample under investigation is prepared. The former sample is made of Brea Sand Stone and has the dimensions of 2.54 by 10.15 centimeters. Nazirdoust et al. have transformed the two dimensional data of the former sample into three dimensional data through the Scanning method. Statistical characteristics of the fracture including average diaphragm and standard deviation have been already calculated in [12]. Fracture diaphragm ranges between 240 microns to 1.2 millimeters. There are no complete barriers in this fracture and therefore, simulation of the two dimensional flow can be performed with least difficulty. In order to put the results of simulation into real applications, certain minimum statistical characteristics of the fracture are required. Average and variance values of fracture opening can be these characteristics. However, investigation of curvatures is harder and is accompanied with more complexities. Authors in [12] have calculated the data regarding average fracture opening (height of plates) $\left(\mathrm{H}_{\mathrm{avg}}\right)$, standard deviation, fracture area and curve area. Curve or bending is defined as percentile increase in traveled distance by the fluid through a fracture resulting from coarseness or hardness of fracture walls. According to the definition provided in [12] curve or bending is calculated as follows:

$$
\theta=\frac{L_{\text {flow }}}{L}-1
$$

In the upper equation, $\mathrm{L}_{\text {flow }}$ is the route that fluid passes through and $L$ is the length of fracture. In physical conditions, it is difficult to measure the amount of bending. Therefore, the lines of flow route along the fracture lines for laminar flows can be measured through the CFD method. In order to measure the bending, a water fluid flow with velocity of $0.1 \mathrm{~m} / \mathrm{s}$ and with at least 4 particles of 10nanometer drops was simulated through the discrete phase modeling method. It is worth mentioning that Brownian effects have been neglected. 
A 155000 cell calculative network has been performed in Ansys and afterwards, fluent method has been used for this simulation. A single-phase water and air flow has been passed through a fracture. A density of $998.2 \mathrm{~kg} / \mathrm{m}^{3}$ and viscosity of $0.001 \mathrm{~kg} / \mathrm{m} \cdot \mathrm{s}$ have been supposed for water. In addition, in case of Air, the former values have been respectively supposed as $1.225 \mathrm{~kg} / \mathrm{m}^{3}$ and $1.8 \times 10^{-5}$. In addition in terms of pressure, a gradient of 1000 to $5 \mathrm{~Pa}$ has been considered for water and a range of 20 to $0.1 \mathrm{~Pa}$ has also been considered for air. Furthermore, permeability of the porous matrix with porosity of $20 \%$ ranges between $0.2 \mathrm{md}$ and $2000 \mathrm{md}$. Nonetheless, a 155000 cell calculative network has been used for this model. Densities and viscosities of water and air are as they're mentioned above. A total 40 simulations have been run for 40 different pressure gradients under single-phase water and air flows.

\section{NETWORK INDEPENDENCE}

In order to guarantee the validity of our solvation, first of all, a network independence test must be performed. The following graph shows the results of investigation of network independence. Determination of a suitable network size consumes the highest amount of time among components of a three dimensional simulation. This is because the quality of network mesh has significant effects on the process of convergence. Numerical solvation of a problem related to fluid dynamics should be network independent. In other words, as the network gets smaller, responses to changes must become more negligible. With respect to the fact that only numerical data are comparable with friction coefficient and in order to find such networks, we are required to draw the graph of changes of friction coefficient according to the number of elements. In this regard, a network with 155000 nodes was established. Calculations have been performed by making use of two parallel computer devices equipped with Intel Core i7 processors and a total memory of 16 gigabytes.

\section{MARGINAL CONDITIONS}

Marginal conditions of input pressure have been made use of for the input that can be seen on the right side of the aforementioned figure. Input pressures have been considered for both the fracture and surrounding matrix. In addition, output pressure has also been considered for both the fracture and its surrounding. Furthermore, a symmetric state has been considered for the upper and lower bounds of the fracture. Remaining surfaces have been considered as solid walls with no flows. The input and out pressures for both the fracture and its surrounding matrix have been considered equally. Nonetheless, a $20 \%$ porous environment has been considered for the matrix.

TABLE I. NETWORK INDEPENDENCE

\begin{tabular}{|c|c|c|c|c|}
\hline $\begin{array}{c}\text { Calculation } \\
\text { time }\end{array}$ & $\begin{array}{c}\text { Friction (water): 10 Pa; } \\
\text { perm.: 2000 md }\end{array}$ & $\begin{array}{c}\text { Friction (Air): 0.2 Pa; } \\
\text { perm.: 2000 md }\end{array}$ & Number of elements & Number of nodes \\
\hline 8 hours & 496.234444 & 8120.498634 & 662329 & 337679 \\
\hline 3 hours & 496.345423 & 8132.873460 & 301995 & 155462 \\
\hline 0.5 hours & 507.98568 & 8796.976339 & 11384 & Medium \\
\hline
\end{tabular}

\section{SimULATION RESUlTS}

Throughout the entire calculations, a constant pressure gradient has been taken into account for surroundings of fractures. As shown, the pressure drop is linear along the flow route (following the Darcy rule). Significant pressure changes are observed near the fracture profile which is due to changes in opening of fracture. Following Figures 1-2 show the changes in pressure for both pressure gradients of $5 \mathrm{~Pa}$ under permeability of $2000 \mathrm{md}$, and $1000 \mathrm{~Pa}$ under permeability of $2000 \mathrm{md}$. As it was expected, fluid flow is more concentrated in the fracture. This has been shown in upper images. The following Figures 3-4 show the flow of air with pressure range of $5 \mathrm{~Pa}$ to $1000 \mathrm{~Pa}$ under a permeability rate of $2000 \mathrm{md}$ and a porosity of $20 \%$. Vectors of velocity show that lower flow rates are associated with surroundings of fractures. This figure reveals the complex relations of fluid flow between fractures and their surrounding matrixes. As it can be seen, the rate of flow in matrixes surrounding fractures is low and in these cases, the fluid moves through the interface between fracture and impermeable matrix. In terms of laminar flow rates, the calculated pressure drop is linear and is comparable with flow rate. Results of simulations have shown that a significant portion of pressure drop in fractures takes place in areas with smallest openings or diaphragms.

Figures 5-6 show the friction coefficient according to flow rate for the steady pressure gradient model for both the water and air flows under different permeability in surrounding matrices. In addition, our simulations have shown that matrix permeability can have significant effects of flow's transfer behavior. As shown in the following diagrams, the low amount of permeability has been diagnosed with the highest friction coefficient in terms of constant flow rates. The relation between coefficient of friction and rate of flow linearly increases along the logarithm diagram and as the flow rate increases, the value of friction coefficient decreases. Table III shows the results of comparison of value of friction coefficient under permeability values of $20 \mathrm{md}$ and $2 \mathrm{md}$. It can be seen that under constant pressures, the friction coefficient of permeability of $2 \mathrm{md}$ is higher than the permeability of $20 \mathrm{md}$. Therefore, it may be concluded that as the permeability is increased, the value of friction coefficient decreases. 

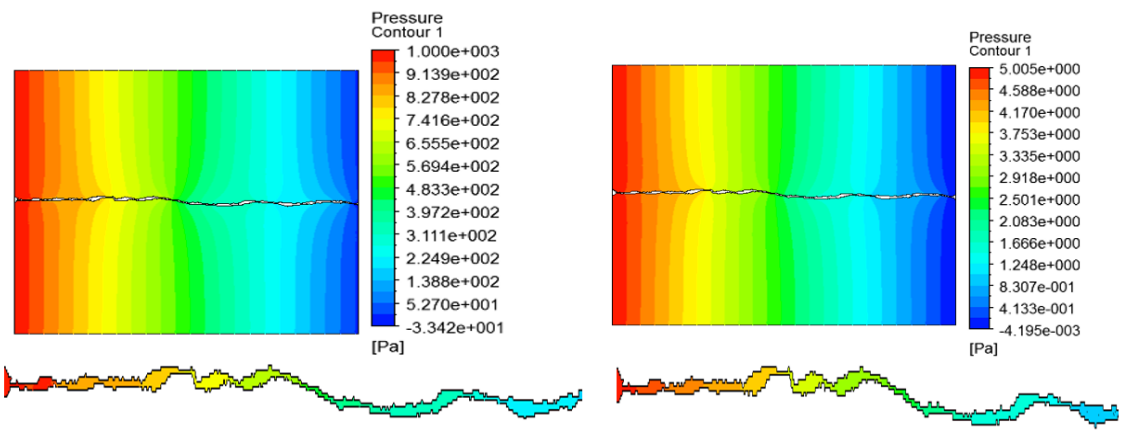

Fig. 1. Factors of pressure gradient under equal permeability for flow of water
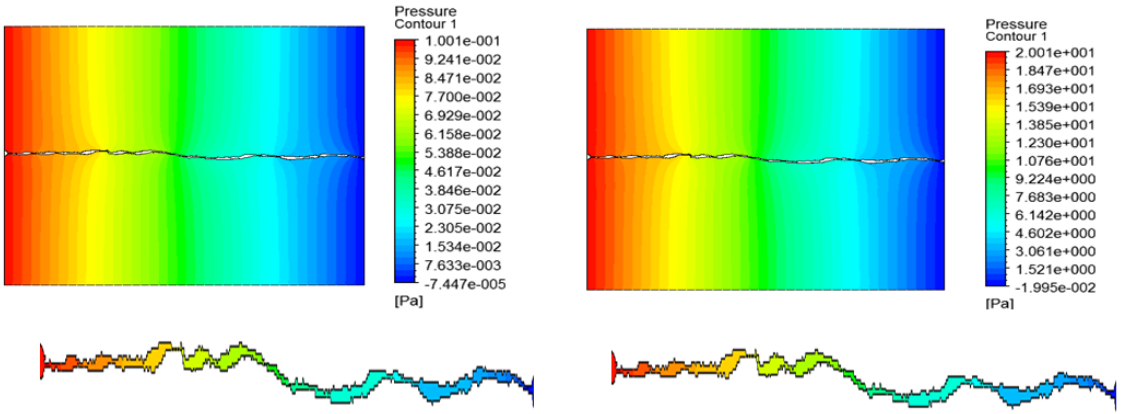

Fig. 2. Factors of pressure gradient under equal permeability for flow of air



Fig. 3. Velocity vector lines under permeability of $2000 \mathrm{md}$ for $5 \mathrm{~Pa}$ air flow



Fig. 4. Velocity vector lines under permeability of $2000 \mathrm{md}$ for $1000 \mathrm{~Pa}$ air flow 


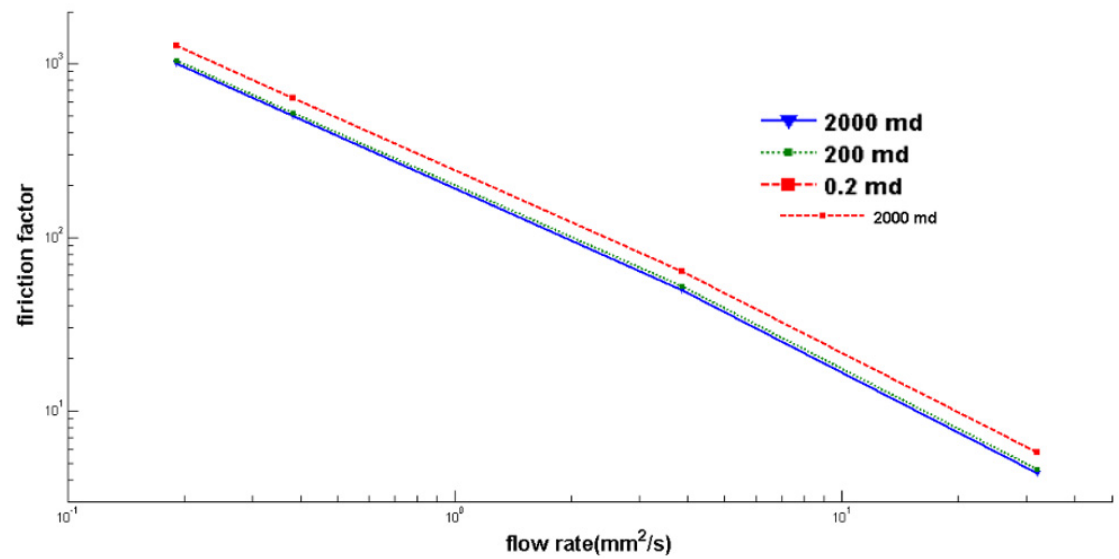

Fig. 5. Diagram of friction coefficient according to flow rate under pressure gradients of 5, 10, 100 and $1000 \mathrm{~Pa}$ and permeability of $0.2,200$ and $2000 \mathrm{md}$ for water flow

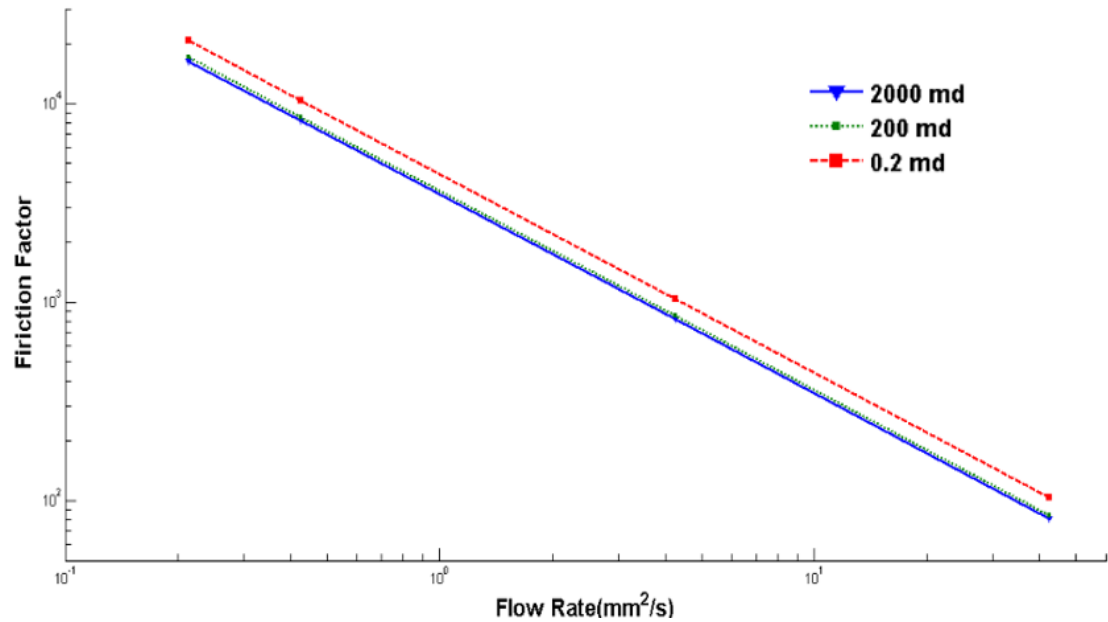

Fig. 6. Diagram of friction coefficient according to flow rate under pressure gradients of 5, 10, 100 and $1000 \mathrm{~Pa}$

TABLE II. INVESTIGATION OF EFFECT OF PERMEABILITY ON FRICTION COEFFICIENT

\begin{tabular}{|c|c|c|c|c|c|c|c|}
\hline Material & Pressure & Flow rate & Reynolds & Permeability & Fric. Coeff. & Permeability & Fric. Coeff. \\
\hline Air & 20 & 42.348 & 2.882 & 20 & 81.305 & 2 & 81.667 \\
\hline Air & 2 & 4.26 & 0.289 & 20 & 816.233 & 2 & 819.846 \\
\hline Air & 0.2 & 0.426 & 0.029 & 20 & 8163.404 & 2 & 8199.635 \\
\hline Air & 0.1 & 0.213 & 0.0145 & 20 & 16313.524 & 2 & 16385.922 \\
\hline Water & 1000 & 33.003 & 31.945 & 20 & 4.382 & 2 & 4.404 \\
\hline Water & 100 & 3.872 & 3.865 & 20 & 49.785 & 2 & 50.008 \\
\hline Water & 10 & 0.383 & 0.382 & 20 & 500.908 & 2 & 503.132 \\
\hline Water & 5 & 0.19174 & 0.191 & 20 & 1003.92 & 2 & 1008.375 \\
\hline
\end{tabular}

\section{CONCLUSIONS \& SUGGESTIONS}

In this paper authors have numerically simulated a singlephase laminar flow with constant pressure gradient through a fractured profile with various permeability rates. Considering the results of the simulations performed in this paper, a relationship has been developed for the evaluation of friction coefficient in fractures surrounded by impermeable matrixes. In addition, this article has proven that the existence of permeable matrices reduces the value of friction coefficient. Considering the permeability factor throughout the fracture has resulted in the creation of a suitable understanding of features or characteristics of flow through fractured areas. Some of the most important conclusions made by this model are:

- The presence of permeable matrices results in reduction of friction coefficient compared to impermeable matrixes

- Highest rates of pressure drop are observed in areas with smaller diaphragms 
- Application of marginal conditions in terms of the relation between fracture and porous matrixes results in an approximate $10 \%$ reduction in simulated flow rate.

Regarding the issues related to the present article, the following could be suggested as future work:

- Investigation of marginal friction, loss of viscosity and other effects including those resulting from orientation, magnetic fields or the effects of radiation in heat transfer in porous matrixes

- Development of methods of flow modeling in porous environments and interfaces of fluids.

\section{REFERENCES}

[1] J. S. Konzuk, B. H. Kueper, "Evaluation of cubic law based models describing single-phase flow through a rough-walled fracture", Water Resources Research, Vol. 40, No. 2, 2004

[2] J. Johnson, S. Brown, "Experimental mixing variablity in intersecting natural fractures", Geophysical Research Letters Vol. 28, No. 22, pp. 4303-4306, 2001

[3] M. Billstein, U. Svensson, N. Johansson, "Application and validation of a numerical model of flow through embankment dams with fractures, comparisons with experimental data", Canadian Geotechnical Journal, Vol. 36, No. 4, pp. 651-659, 1999
[4] R. W. Zimmerman, G. S. Bodvarsson, "Hydraulic conductivity of rock fractures", Transport in Porous Media, Vol. 23, No. 1, pp. 1-30, 1996

[5] A. E. Scheidegger, Physics of Flow through Porous Media, University of Toronto Press, Toronto, 1974

[6] V. Muralidharan, D. Chakravarthy, E. Putra, D. S. Schechter, "Simulation of Fluid Flow Through Rough Fractures", SPE Annual Technical Conference and Exhibition, pp. 26-29, 2004

[7] M. Lespinasse, J. Sausse, "Quantification of fluid flow: hydromechanical behaviour of different natural rough fractures", Journal of Geochemical Exploration, Vol. 69-70, pp. 483-486, 2000

[8] H. H. Liu, C. B. Haukwa, C. F., Ahlers, G. S. Bodvarsson, A. L. Flint, W. B. Guertal, "Model flow and transport in unsaturated fractured rock: an evaluation of the continuum approach", Journal of Contaminant Hydrology, Vol. 62-63, pp. 173-188, 2003

[9] S. L. Bryant, R. K. Paruchuri, K. P. Saripalli, "Flow and solute transport around injection wells through a single growing fracture", Advances in Water Resources, Vol. 26, No. 8, pp. 803-813, 2003

[10] T. Hirono, M. Takahashi, S. Nakashima, "In situ visualization of fluid flow image within deformed rock by X-ray CT", Engineering Geology Vol. 70, No. 1-2, pp. 37-46, 2000

[11] Z. T. Karpyn, G. Li, P. M. Halleck, A. S. Grader, "Factors favoring the formation of fluid banks during counter-current flow in porous media", EGS-AGU-EUG Joint Assembly, 2003

[12] K. Nazridoust, G. Ahmadi, D. H. Smith, "New friction factor correlation for laminar, single phase flows through rock fractures", Journal of Hydrology, Vol. 329, No. 1-2, pp. 315-328, 2006 